53 (2021) pp. 157-168

DOI: https://doi.org/10.33039/ami.2021.02.005

URL: https://ami.uni-eszterhazy.hu

\title{
Dealing with uncertainty: A rough-set-based approach with the background of classical logic*
}

\author{
Tamás Kádek, Tamás Mihálydeák \\ University of Debrecen, Faculty of Informatics \\ tamas.kadek@inf.unideb.hu \\ mihalydeak@unideb.hu \\ Submitted: December 22, 2020 \\ Accepted: February 17, 2021 \\ Published online: May 18, 2021
}

\begin{abstract}
The representative-based approximation has been widely studied in rough set theory. Hence, rough set approximations can be defined by the system of representatives, which plays a crucial role in set approximation. In the authors' previous research a possible use of the similarity-based rough set in first-order logic was investigated. Now our focus has changed to representative-based approximation systems. In this article the authors show a logical system relying on representative-based set approximation. In our approach a three-valued partial logic system is introduced. Based on the properties of the approximation space, our theorems prove that in some cases, there exists an efficient way to evaluate the first-order formulae.
\end{abstract}

Keywords: Rough set theory, set approximation, approximation-based logic system

AMS Subject Classification: 03E72

\section{Introduction}

Nowadays a huge amount of data appear in an information system and they have to be treated in order to get new information, to make decisions, etc. Behind the

${ }^{*}$ This work was supported by the construction EFOP-3.6.3-VEKOP-16-2017-00002. The project was supported by the European Union, co-financed by the European Social Fund. 
data there are objects with (probably different) properties. Properties are handled in two steps: as attributes and the corresponding attribute values. In the real practice finite number of attributes and that of the corresponding attribute values can be used. Usually, there are more objects than combination of attribute values, therefore more than one objects are represented by the same attribute values, and so they are indiscernible relying on the background knowledge embedded in an information system. Indiscernible objects have to be treated in the same way.

Pawlak's original system of rough sets shows the consequences of indiscernibility [10-12]. In many practical cases not only indiscernible objects have to be treated in the same way, but objects with the same attribute values of some (and not all) attributes. This is one of the theoretical bases of the generalizations of Pawlak's original theory. In rough sets theory the objects to be treated in the same way belong to a base set. Informally in granular computing a granule contains objects which have to be treated in the same way. Granules play - as the most fundamental concept - a crucial role in granular computing, it means that granules (and not objects belonging to them) are in the focus of investigations.

Representatives are used for representing a whole group of objects. In a very general case to choose representatives (granules) is not a trivial problem. In the case of a system relying on an indiscernible relation, any object can represent the corresponding indiscernible set of objects. When a tolerance relation is used, then the method of correlation clustering gives a possibility to define representatives (see $[1,8])$. Based on the different techniques to find representatives some generalization of the approximation space must be considered.

From the logical point of view, a natural question arises: is there any possibility to create a first-order logical system relying on representatives? If the answer is yes, then the consequence relation can be used in order to get (or check) new information. In this paper the authors define first-order logical semantics and show some of its important properties.

Logical systems based on rough sets are also widely studied [9], so it seems easy to predict the results, but the investigation should repeat when a new viewpoint appears. In this work we will use the most recent general definition of representativebased approximation space. The main goal is to define a logical system which uses only the representatives when a decision about a certain group of objects is made.

The structure of the paper is the following: at first, we will define the representative-based approximation system, where instead of base sets the extension of representatives is used. Then a one-argument first-order language is introduced with approximation-space-based semantics. We will show how to generate approximative interpretations from an existing classical one. Finally, the key properties of our system will be discovered with the help of a few theorems.

\section{Representative-based approximation spaces}

Definition 2.1. The triple $\langle U, R, \mathfrak{R}\rangle$ is a representative-based approximation space if 
1. $U$ is a nonempty set of objects,

2. $R=\left\{r_{1}, r_{2}, \ldots, r_{k}\right\}$ where $k \geq 1$ is a set of representatives,

3. $\mathfrak{R} \subseteq R \times U$ is a relation.

Definition 2.2. Let $r_{i}$ be a representative, i.e. $r_{i} \in R$. Then

$$
\left\langle\left\langle r_{i}\right\rangle\right\rangle^{\langle U, R, \mathfrak{R}\rangle}=\left\{u: r_{i} \mathfrak{R} u\right\}
$$

is the extension of $r_{i}$. We shortly will write $\left\langle\left\langle r_{i}\right\rangle\right\rangle$ if it does not cause any misunderstanding.

There exists a general agreement to restrict $\left\langle\left\langle r_{i}\right\rangle\right\rangle$ at least saying that it shall not be empty, but this constraint is now unnecessary. Although is straightforward that a representative with empty extension can not be useful during the approximation.

Definition 2.3. The approximation pair $\langle\mathfrak{l}, \mathfrak{u}\rangle$ of the representative-based approximation space $\langle U, R, \mathfrak{R}\rangle$ is a pair of mappings $2^{U} \rightarrow 2^{U}$ defined as follows

$$
\begin{aligned}
\mathfrak{l}(S) & =\cup\left\{\left\langle\left\langle r_{i}\right\rangle\right\rangle: r_{i} \in R \text { and }\left\langle\left\langle r_{i}\right\rangle\right\rangle \subseteq S\right\} ; \\
\mathfrak{u}(S) & =\cup\left\{\left\langle\left\langle r_{i}\right\rangle\right\rangle: r_{i} \in R \text { and }\left\langle\left\langle r_{i}\right\rangle\right\rangle \cap S \neq \emptyset\right\} .
\end{aligned}
$$

From this point, the $\left\langle\left\langle r_{i}\right\rangle\right\rangle$ extensions of the representatives can be considered as base sets of a union-type approximation space [2].

Definition 2.4. Let $\langle U, R, \Re\rangle$ be a representative-based approximation space and $u \in U$. Then the representative vector of $u$ (denoted by $[u]^{\langle U, R, \Re\rangle}$ or simply $[u]$ if it does not cause any misunderstanding) is the following:

$$
\begin{aligned}
{[u]^{\langle U, R, \Re\rangle} } & =\left\langle[u]_{1}^{\langle U, R, \Re\rangle}, \ldots,[u]_{k}^{\langle U, R, \Re\rangle}\right\rangle \text { where } \\
{[u]_{i}^{\langle U, R, \Re\rangle} } & = \begin{cases}1 & \text { if } u \in\left\langle\left\langle r_{i}\right\rangle\right\rangle, \\
0 & \text { otherwise, }\end{cases}
\end{aligned}
$$

Some common properties of the approximation space can be determined by analyzing the representative vectors:

$$
\sigma(u)=\sum_{i=1}^{k}[u]_{i}
$$

- if $\sigma(u)=1$ for all $u \in U$, then the approximation space is based on a partition generated by $\mathfrak{R}$;

- if $\sigma(u)=0$ for some $u \in U$, then the approximation space is partial, because $u$ is an object without any representative, and so $u \notin \mathfrak{l}(S)$ and $u \notin \mathfrak{u}(S)$ for all $S \subseteq U$;

- if $\sigma(u) \geq 2$ for some $u \in U$, then the approximation space contains overlapping (not disjoint) extensions for some representatives. See more about covering systems relying on tolerance relations in [14] and about general covering systems in $[13,15]$. 


\section{One-argument first-order language}

We begin the investigation with a simplified first-order language which allows oneargument predicate parameters only. The simplified language could be easily extended with other predicate parameters [7], and it is expressive enough for further investigations [3].

Definition 3.1. The ordered 4-tuple $\langle L C$, Var, Pred,Form $\rangle$ is a one-argument first-order language containing only one-argument predicate parameters if

1. $L C=\{\neg, \wedge, \vee, \supset, \exists, \forall,()$,$\} is the set of logical constants;$

2. $\operatorname{Var}=\left\{x_{1}, x_{2}, \ldots\right\}$ is a countably infinite set of variables;

3. Pred is a nonempty set of one-argument predicate parameters;

4. LC, Var, and Pred are pairwise disjoint;

5. the set of formulae denoted by Form is defined inductively:

(a) if $P \in$ Pred and $x \in \operatorname{Var}$, then $P(x) \in$ Form and is an atomic formula,

(b) if $A \in$ Form, then $\neg A \in$ Form,

(c) if $A, B \in$ Form and $\circ \in\{\wedge, \vee, \supset\}$, then $(A \circ B) \in$ Form,

(d) if $A \in$ Form and $x \in V$ Var, then $\exists x A \in$ Form and $\forall x A \in$ Form.

\subsection{Interpretation}

The conventional Aristotelian semantics of a one-argument first-order language is very widely known, hence it is not introduced here, only the interpretation of the language is recalled.

Definition 3.2. The pair $\langle U, \psi\rangle$ is an interpretation of the one-argument first-order language $\langle L C$, Var, Pred, Form $\rangle$ if

1. $U$ is a nonempty set of objects,

2. $\psi$ is a mapping Pred $\rightarrow 2^{U}$.

In the classical first-order logic, if $\langle U, \psi\rangle$ is an interpretation on a given $U$ set of objects, and $P$ is a one-argument predicate parameter of the language, then the semantic value of $P$ is usually given as $\psi(P) \subseteq U$ :

- $u \in \psi(P)$ means that $u$ belongs to the positivity domain of $P$, or we can say that $P$ is true on $u$,

- $u \in U \backslash \psi(P)$ means that $u$ belongs to the negativity domain of $P$, or we can say that $P$ is false on $u$. 
Next, we define the semantics of a one-argument first-order language with the help of a representative-based approximation space. The idea is to approximate the positivity and negativity domains adapting the solution explained in [6]. To do so, first we introduce the representative-based approximative interpretation.

Definition 3.3. The ordered 4-tuple $\langle U, R, \Re, \varrho\rangle$ is an approximative interpretation of the one-argument first-order language $\langle L C$, Var, Pred, Form $\rangle$ if

1. $\langle U, R, \Re\rangle$ is a representative-based approximation space,

2. $\varrho$ is a mapping such that $\varrho(P)=\left\langle\varrho(P)_{1}, \ldots, \varrho(P)_{k}\right\rangle$ for all $P \in$ Pred, where

(a) $\varrho(P)_{\ell} \in\{-1,0,1\}(\ell=1, \ldots, k)$; and

(b) there is no $u \in U$ and $i, j \in\{1, \ldots, k\}$ such that

$$
[u]_{i} \cdot \varrho(P)_{i}=1 \text { and }[u]_{j} \cdot \varrho(P)_{j}=-1
$$

where $k$ is the number of representatives, hence $R=\left\{r_{1}, \ldots, r_{k}\right\}$.

The $\varrho(P)_{i}$ represents the relationship between the $i$ th representative $\left(r_{i}\right)$ and the semantic value of the one-argument predicate $P$ :

- if $\varrho(P)_{i}=+1$, then $r_{i}$ certainly belongs to the positivity domain of $P$;

- if $\varrho(P)_{i}=-1$, then $r_{i}$ certainly belongs to the negativity domain of $P$;

- if $\varrho(P)_{i}=0$, then we cannot decide whether $r_{i}$ belongs to the positivity domain or not. We could say that $r_{i}$ is in the boundary region.

The arithmetic product $[u]_{i} \varrho \varrho(P)_{i}$ is used to express the connection between an arbitrary object $u \in U$ and the semantic value of $P$ with the help of the $i$ th representative. Our definition excludes the contradiction when different representatives of $u$ belong certainly to the positivity and negativity domain of $P$. Now we show a method to satisfy this condition with the help of an interpretation.

Definition 3.4. Let $\langle U, R, \mathfrak{R}\rangle$ be a representative-based approximation space, $\mathcal{L}$ be a one-argument first-order language, and $\langle U, \psi\rangle$ be its interpretation. The

$$
\varrho(P)_{i}=\left\{\begin{aligned}
1 & \text { if }\left\langle\left\langle r_{i}\right\rangle\right\rangle \subseteq \psi(P), \\
-1 & \text { if }\left\langle\left\langle r_{i}\right\rangle\right\rangle \cap \psi(P)=\emptyset, \\
0 & \text { otherwise; }
\end{aligned}\right.
$$

function is the derived mapping from $\psi$ with respect to a given $\langle U, R, \mathfrak{R}\rangle$.

Theorem 3.5. Let $\langle U, R, \mathfrak{R}\rangle$ be a representative-based approximation space, $\mathcal{L}$ be a one-argument first-order language, and $\langle U, \psi\rangle$ be its interpretation. If $\varrho$ is the derived mapping from $\psi$ with respect to $\langle U, R, \Re\rangle$, then there is no $u \in U$ and $i, j \in\{1, \ldots, k\}$ such that $[u]_{i} \varrho \varrho(P)_{i}=1$ and $[u]_{j} \cdot \varrho(P)_{j}=-1$. 
Proof. If $[u]_{i} \cdot \varrho(P)_{i}=1$ for some $u \in U$ and $i \in\{1, \ldots, k\}$, then both $[u]_{i}=1$ and $\varrho(P)_{i}=1$. By definition, $[u]_{i}=1$ when $u \in\left\langle\left\langle r_{i}\right\rangle\right\rangle$ and $\varrho(P)_{i}=1$ when $\left\langle\left\langle r_{i}\right\rangle\right\rangle \subseteq \psi(P)$, so $u \in \psi(P)$. Indirectly supposing that there exists a $j \in\{1, \ldots, k\}$ such that $[u]_{j} \varrho \varrho(P)_{j}=-1$, the following contradiction appears: $[u]_{j}=1$, so $u \in\left\langle\left\langle r_{j}\right\rangle\right\rangle$, which means that $\left\langle\left\langle r_{j}\right\rangle\right\rangle \cap \psi(P) \neq \emptyset$, but $\varrho(P)_{j}=-1$, hence the previous intersection should be empty.

Corollary 3.6. Let $\langle U, R, \Re\rangle$ be a representative-based approximation space, $\mathcal{L}$ be a one-argument first-order language, $\langle U, \psi\rangle$ be its interpretation, and $\varrho$ be the derived mapping from $\psi$. Then $\langle U, R, \Re, \varrho\rangle$ is an approximative interpretation.

The value of $\varrho(P)_{i}$ - if it is derived from the $\langle U, \psi\rangle$ interpretation - shows the relationship between the positivity domain of $P$ and extension $\left\langle\left\langle r_{i}\right\rangle\right\rangle$ of the $i$ th representative:

- If $\varrho(P)_{i}=1$, then all members of the extension of $r_{i}$ (all objects represented by $r_{i}$ ) are in the positiviy domain of $P$; $P$ is certainly true for all $u \in\left\langle\left\langle r_{i}\right\rangle\right\rangle$.

- If $\varrho(P)_{i}=-1$, then all members of the extension of $r_{i}$ are in the negativity domain of $P$; $P$ is certainly false for all $u \in\left\langle\left\langle r_{i}\right\rangle\right\rangle$.

- If $\varrho(P)_{i}=0$, then some members of the extension of $r_{i}$ belong to the positivity domain, while others belong to the negativity domain.

\subsection{Semantics}

A widely used technique in rough set theory is to distinguish between optimistic and pessimistic approaches [7]. At this point it is crucial to analyze the information about objects, especially in the case when different representatives declare different facts about the positivity and negativity domain of a predicate.

The tables in Fig. 1 summarize the difference of four approaches. The heads of the tables contain the maximum and the rows contain the minimum of the set:

$$
\Delta(P, u)=\left\{\varrho(P)_{i}: i \in\{1, \ldots, k\},[u]_{i}=1\right\}
$$

The bottom left corners are empty hence this kind of contradiction was not allowed in Definition 3.3. If $\Delta \neq \emptyset$, when $u$ has at least one representative, then the following approaches appear:

1. Optimistic approach: we take the maximum of $\Delta(P, u)$, so if there exists at least one representative of $u$ that belongs to the positivity domain of $P$, we will suppose that $P$ is true on $u$.

2. Pessimistic approach: we take the minimum of $\Delta(P, u)$, so we suppose that $P$ is true on $u$ only if all the representatives of $u$ belong to the positivity domain of $P$. 
3. Union-based approach: we say that $u$ belongs to the union of its representatives. This implies that if at least one representative belongs to the border, then we cannot say anything certain about $u$.

4. Intersection-based approach: we say that $u$ belongs to the intersection of its representatives. This implies that uncertainty will appear only if all the representatives of $u$ belong to the border.

\begin{tabular}{c|lll}
$\Delta(P, u)$ & 1 & 0 & -1 \\
\hline 1 & 1 & & \\
0 & 1 & 0 & \\
-1 & & 0 & -1
\end{tabular}

Optimistic Approach

\begin{tabular}{c|lll}
$\Delta(P, u)$ & 1 & 0 & -1 \\
\hline 1 & 1 & & \\
0 & 0 & 0 & \\
-1 & & 0 & -1
\end{tabular}

Union-Based Approach

\begin{tabular}{c|rrr}
$\Delta(P, u)$ & 1 & 0 & -1 \\
\hline 1 & 1 & & \\
0 & 0 & 0 & \\
-1 & & -1 & -1
\end{tabular}

Pessimistic Approach

\begin{tabular}{c|rrr}
$\Delta(P, u)$ & 1 & 0 & -1 \\
\hline 1 & 1 & & \\
0 & 1 & 0 & \\
-1 & & -1 & -1
\end{tabular}

Intersection-Based Approach

Figure 1. Managing contradicting information.

By respecting the set theoretic view of the extension of representatives (introduced in Definition 2.2 and also used later in Definition 3.4), it is a straightforward decision to adopt the intersection-based approach.

Definition 3.7. Let $\langle U, R, \Re, \varrho\rangle$ be an approximative interpretation. The function $v: \operatorname{Var} \rightarrow U$ is an assignment relying on the approximative interpretation.

Definition 3.8. Let $v$ be an assignment relying on the $\langle U, R, \Re, \varrho\rangle$ approximative interpretation. The assignment $v[x: u]$ denotes a modified assignment which is defined as follows:

$$
v[x: u](y)= \begin{cases}u & \text { if } y=x, \\ v(y) & \text { otherwise. }\end{cases}
$$

Note that we defined the assignment and the modified assignment exactly in the same way as it was introduced in the classical first-order logic. It helps us to compare the evaluation method later.

Definition 3.9. The semantic value of $P \in$ Pred is the following

$$
U \rightarrow\left\{0,{ }^{1} / 2,1\right\} \cup\{2\}
$$


function:

$$
\llbracket P \rrbracket^{\langle U, R, \Re, \varrho\rangle}(u)= \begin{cases}2 & \text { if }[u]_{i}=0 \text { for all } i \in\{1, \ldots, k\} \\ 1 & \text { if }[u]_{i} \cdot \varrho(P)_{i}=1 \text { for some } i \in\{1, \ldots, k\} \\ 0 & \text { if }[u]_{i} \cdot \varrho(P)_{i}=-1 \text { for some } i \in\{1, \ldots, k\} \\ 1 / 2 & \text { otherwise. }\end{cases}
$$

As a consequence of the system's possible partiality, logic with truth value gap is used. The value 2 represents the lack of truth value.

Theorem 3.10. Let $\langle L C, V$ ar, Pred,Form $\rangle$ be a one-argument first-order language and $\langle U, R, \Re, \varrho\rangle$ be its approximative interpretation relying on the representative-based approximation space $\langle U, R, \Re\rangle$ where $\varrho$ is the derived mapping from $\psi$; then $\llbracket P \rrbracket(u)=1$ if and only if $u \in \mathfrak{l}(\psi(P))$ for all $u \in U$.

Proof. Let us create the proof in two steps:

1. If $\llbracket P \rrbracket(u)=1$ then there exists an $r_{i} \in R$ such that $[u]_{i} \cdot \varrho(P)_{i}=1$ and so $u \in\left\langle\left\langle r_{i}\right\rangle\right\rangle$ (based on Definition 2.4) and $\left\langle\left\langle r_{i}\right\rangle\right\rangle \subseteq \psi(P)$ (based on Definition 3.4). When $\left\langle\left\langle r_{i}\right\rangle\right\rangle \subseteq \psi(P)$ then $\left\langle\left\langle r_{i}\right\rangle\right\rangle \subseteq \mathfrak{l}(\psi(P))$ and so $u \in \mathfrak{l}(\psi(P))$.

2. If $u \in \mathfrak{l}(\psi(P))$ then there exists an $r_{i}$ such that $u \in\left\langle\left\langle r_{i}\right\rangle\right\rangle$ and $\left\langle\left\langle r_{i}\right\rangle\right\rangle \subseteq \psi(P)$ and so $[u]_{i}=1$ and $\varrho(P)_{i}=1$ therefore $\llbracket P \rrbracket(u)=1$ hence $[u]_{i} \cdot \varrho(P)_{i}=1$.

The idea to use a partial three-valued system appeared in $[4,7]$.

Definition 3.11. The semantic value of the formula $A \in$ Form using the interpretation $\langle U, R, \mathfrak{R}, \varrho\rangle$ is denoted by $\llbracket A \rrbracket_{v}^{\langle U, R, \Re, \varrho\rangle}$ or simply $\llbracket A \rrbracket_{v}$ and defined as follows:

$$
\begin{aligned}
& \llbracket P(x) \rrbracket_{v}=\llbracket P \rrbracket(v(x)) \\
& \llbracket \neg A \rrbracket_{v}= \begin{cases}2 & \text { if } \llbracket A \rrbracket_{v}=2 \\
1-\llbracket A \rrbracket_{v} & \text { otherwise; }\end{cases} \\
& \llbracket(A \wedge B) \rrbracket_{v}= \begin{cases}2 & \text { if } \llbracket A \rrbracket_{v}=2 \text { or } \llbracket B \rrbracket_{v}=2 \\
\min \left\{\llbracket A \rrbracket_{v}, \llbracket B \rrbracket_{v}\right\} & \text { otherwise; }\end{cases} \\
& \llbracket(A \vee B) \rrbracket_{v}= \begin{cases}2 & \text { if } \llbracket A \rrbracket_{v}=2 \text { or } \llbracket B \rrbracket_{v}=2 \\
\max \left\{\llbracket A \rrbracket_{v}, \llbracket B \rrbracket_{v}\right\} & \text { otherwise; }\end{cases} \\
& \llbracket(A \supset B) \rrbracket_{v}= \begin{cases}2 & \text { if } \llbracket A \rrbracket_{v}=2 \text { or } \llbracket B \rrbracket_{v}=2 \\
\max \left\{1-\llbracket A \rrbracket_{v}, \llbracket B \rrbracket_{v}\right\} & \text { otherwise; }\end{cases}
\end{aligned}
$$

Let $\mathcal{V}=\left\{u: u \in U\right.$ and $\left.\llbracket A \rrbracket_{v[x: u]} \neq 2\right\}$.

$$
\llbracket \exists x A \rrbracket_{v}= \begin{cases}2 & \text { if } \mathcal{V}=\emptyset, \\ \max _{u \in \mathcal{V}}\left\{\llbracket A \rrbracket_{v[x: u]}\right\} & \text { otherwise; }\end{cases}
$$




$$
\llbracket \forall x A \rrbracket_{v}= \begin{cases}2 & \text { if } \mathcal{V}=\emptyset \\ \min _{u \in \mathcal{V}}\left\{\llbracket A \rrbracket_{v[x: u]}\right\} & \text { otherwise; }\end{cases}
$$

Like in the classical case, $\exists$ and $\forall$ quantifiers are defined as the generalizations of $\vee$ and $\wedge$, respectively.

\section{Key properties of the approximation}

Theorem 4.1. Let $\mathcal{L}=\langle L C$, Var, Pred,Form $\rangle$ be a one-argument first-order language and $I=\langle U, R, \Re, \varrho\rangle$ be an approximative interpretation of $\mathcal{L}$. There exists an approximative interpretation $J=\left\langle U^{\prime}, R, \mathfrak{R}^{\prime}, \varrho\right\rangle$ such that

$$
\left|U^{\prime}\right| \leq 2^{k} \text { and } \llbracket A \rrbracket_{v}^{I}=\llbracket A \rrbracket_{w}^{J} \text { for all } A \in \text { Form }
$$

where $w(x)=\tau(v(x))$ for some mapping $\tau: U \rightarrow U^{\prime}$.

Proof. We present a construction for such an interpretation $J=\left\langle U^{\prime}, R, \Re^{\prime}, \varrho\right\rangle$ and mapping $\tau$ :

$$
\begin{aligned}
\tau(u) & =\sum_{i=1}^{k} 2^{k-1}[u]_{i}^{\langle U, R, \mathfrak{R}\rangle} \\
U^{\prime} & =\{\tau(u): u \in U\} \\
\mathfrak{R}^{\prime} & =\left\{\left\langle r_{i}, \tau(u)\right\rangle:\left\langle r_{i}, u\right\rangle \in \mathfrak{R}\right\} .
\end{aligned}
$$

It is clear that $U^{\prime} \subseteq\left\{0,1, \ldots, 2^{k}-1\right\}$ so the cardinality condition of $U^{\prime}$ is satisfied. Because of the definition of the $\mathfrak{R}^{\prime}$ relation,

$$
[u]_{i}^{\langle U, R, \Re\rangle}=[\tau(u)]_{i}^{\left\langle U^{\prime}, R, \Re^{\prime}\right\rangle} \text { therefore } \llbracket P \rrbracket^{I}(v(x))=\llbracket P \rrbracket^{J}(w(x))
$$

so the theorem is proved for atomic formulae and can be proved for arbitrary formulae with the help of structural induction.

Corollary 4.2. During the evaluation process of a quantified formula, instead of using all members of the set $U$, it is enough to consider $2^{k}$ objects only. It can dramatically increase the speed of quantified formulae evaluation and so it can reduce the computation time.

\subsection{Properties of the approximation on covering systems}

Theorem 4.3. The one-argument first-order language $\langle L C$, Var, Pred, Form $\rangle$ and its approximative interpretation $\langle U, R, \Re, \varrho\rangle$ generate a three-valued logic system without truth value gap if $\langle U, R, \Re\rangle$ is a covering approximation space. 
Proof. In case of an arbitrary atomic formula $P(x)$ and an arbitrary approximative interpretation $\langle U, R, \Re, \varrho\rangle$, truth value gap (2) can appear as the semantic value of $\llbracket P(x) \rrbracket_{v}$ only if $\sigma(v(x))=0$, but in a covering approximation space $\sigma(u)>0$ for all $u \in U$. So the theorem is proved for atomic formulae and can be proved for arbitrary formulae with the help of structural induction.

Theorem 4.4. Let $\langle L C, V$ ar, Pred, Form $\rangle$ be a one-argument first-order language and $\langle U, R, \mathfrak{R}, \varrho\rangle$ be its approximative interpretation relying on the representativebased covering approximation space $\langle U, R, \Re\rangle$ where $\varrho$ is the derived mapping from $\psi$, and let $v$ be an arbitrary assignment.

$$
\text { If } \llbracket A \rrbracket_{v}^{\langle U, R, \Re, \varrho\rangle} \in\{0,1\} \text { then } \llbracket A \rrbracket_{v}^{\langle U, R, \Re, \varrho\rangle}=|A|_{v}^{\langle U, \psi\rangle} .
$$

Proof. First we will show, that the statement is true for any arbitrary atomic formula:

- If $\llbracket P(x) \rrbracket_{v}=1$ then $[v(x)]_{i} \cdot \varrho(P)_{i}=1$ for some $i \in\{1, \ldots, k\}$.

$-v(x) \in\left\langle\left\langle r_{i}\right\rangle\right\rangle$ because of $[v(x)]_{i}=1$

(as a consequence of Definition 2.4);

- $\left\langle\left\langle r_{i}\right\rangle\right\rangle \subseteq \psi(P)$ hence $\varrho(P)_{i}=1$ and $\varrho$ is derived from $\psi$ (see Definition 3.4);

therefore $v(x) \in \psi(P)$ so $|P(x)|_{v}=1$.

- If $\llbracket P(x) \rrbracket_{v}=0$ then $[v(x)]_{i} \cdot \varrho(P)_{i}=-1$ for some $i \in\{1, \ldots, k\}$.

$-v(x) \in\left\langle\left\langle r_{i}\right\rangle\right\rangle$ because of $[v(x)]_{i}=1$;

- $\left\langle\left\langle r_{i}\right\rangle\right\rangle \cap \psi(P)=\emptyset$ hence $\varrho(P)_{i}=-1$ and $\varrho$ is derived from $\psi$;

therefore $v(x) \notin \psi(P)$ so $|P(x)|_{v}=0$.

Now the theorem can be proved by using structural induction which is trivial in case of zero order connectives and very similar in case of the quantifiers, therefore we focused on the existentially quantified expressions only. Supposing that the theorem is true for the formula $A$ :

- $\llbracket \exists x A \rrbracket_{v}=1$ guarantees that there exists a $v[x: u]$ modified assignment so that $\llbracket A \rrbracket_{v[x: u]}=1$. As we have above supposed, $|A|_{v[x: u]}=1$ so $|\exists x A|_{v}=1$.

- $\llbracket \exists x A \rrbracket_{v}=0$ implies that $\llbracket A \rrbracket_{v[x: u]}=0$ for all $v[x: u]$ modified assignment. Therefore $|A|_{v[u: x]}=0$ for all $u \in U$ so $|\exists x A|_{v}=0$. Remember that $\llbracket A \rrbracket_{v[x: u]} \neq 2$ if the approximative interpretation relies on a covering approximation space as it was proved in Theorem 4.3. 


\section{Conclusion and future work}

In this article we have successfully shown a possible semantic background of a one-argument first-order logical system based on a representative-based approximation. An approximative interpretation was introduced, which we can derive from a classical first-order interpretation easily. We have compared the classical and the approximation-based evaluation, and we have found that, at least in the case of a covering approximation space, we can predict the semantic value of a formula by using the approximation.

Thanks to the promising results shown in the theorems, we have the theoretical basis for further investigations. One possible direction is to analyze the logical laws and inference schemes of the first-order logic in case of different granule systems. The investigation will follow the methods presented in $[3,5]$. We hope that the result of the planned research could be a calculus over a three-valued partial system relying on the representative-based approximation space.

\section{References}

[1] L. Aszalós, T. Mihálydeák: Rough Clustering Generated by Correlation Clustering, in: Rough Sets, Fuzzy Sets, Data Mining, and Granular Computing, Springer Berlin Heidelberg, 2013, pp. 315-324, DOI: https://doi.org/10.1109/TKDE.2007.1061.

[2] Z. E. Csajbóк, T. Minálydeák: General set approximation and its logical applications, in: Jan. 2015, pp. 33-40, DOI: https://doi.org/10.14794/ICAI.9.2014.1.33.

[3] T. Kádek, T. Mihálydeák: On (in)Validity of Aristotle's Syllogisms Relying on Rough Sets, Annals of Computer Science and Information Systems 7 (2015), pp. 35-40.

[4] T. MinÁlydé́k: First-Order Logic Based on Set Approximation: A Partial Three-Valued Approach, in: 2014 IEEE 44th International Symposium on Multiple-Valued Logic, May 2014, pp. 132-137,

DOI: https://doi.org/10.1109/ISMVL. 2014.31.

[5] T. Minálydéx: Aristotle's Syllogisms in Logical Semantics Relying on Optimistic, Average and Pessimistic Membership Functions, in: Rough Sets and Current Trends in Computing: 9th International Conference, RSCTC 2014, Granada and Madrid, Spain, July 9-13, 2014. Proceedings, ed. by C. Cornelis, M. Kryszkiewicz, D. Ślęzak, E. M. Ruiz, R. Bello, L. Shang, Cham: Springer International Publishing, 2014, pp. 59-70, ISBN: 978-3-319-086446 , DOI: http://dx.doi.org/10.1007/978-3-319-08644-6_6.

[6] T. Minálydeák: Logic on Similarity Based Rough Sets, in: Rough Sets, ed. by H. S. Nguyen, Q.-T. Ha, T. Li, M. Przybyєa-Kasperek, Cham: Springer International Publishing, 2018, pp. 270-283, ISBN: 978-3-319-99368-3.

[7] T. Minálydeák: Partial First-Order Logical Semantics Based on Approximations of Sets, Non-classical Modal and Predicate Logics (2011), ed. by M. V. Petr Cintula Shier Ju, pp. 85-90.

[8] D. Nagy, T. Mihálydeák, L. Aszalós: Similarity Based Rough Sets, in: Rough Sets: International Joint Conference, IJCRS 2017, Olsztyn, Poland, July 3-7, 2017, Proceedings, Part II, ed. by L. Polkowski, Y. Yao, P. Artiemjew, D. Ciucci, D. Liu, D. Ślęzak, B. Zielosko, Cham: Springer International Publishing, 2017, pp. 94-107, ISBN: 978-3-31960840-2,

DOI: https://doi.org/10.1007/978-3-319-60840-2_7. 
[9] P. Pagliani, M. Chakraborty: Logic and Rough Sets: An Overview, in: A Geometry of Approximation: Rough Set Theory: Logic, Algebra and Topology of Conceptual Patterns, Dordrecht: Springer Netherlands, 2008, pp. 169-191, ISBN: 978-1-4020-8622-9, DOI: https://doi.org/10.1007/978-1-4020-8622-9_5.

[10] Z. Pawlak: Rough Sets: Theoretical Aspects of Reasoning about Data, Theory and Decision Library D: Springer Netherlands, 1991, ISBN: 9780792314721, URL: https://books.google.hu/books?id=MJPLCqIniGsC.

[11] Z. PAWlaK: Rough sets, International Journal of Computer \& Information Sciences 11.5 (1982), pp. 341-356, ISsN: 1573-7640, DOI: http://dx.doi.org/10.1007/BF01001956.

[12] Z. Pawlak, A. Skowron: Rough sets and Boolean reasoning, Information sciences 177.1 (2007), pp. 41-73.

[13] Z. Pawlak, A. Skowron: Rudiments of rough sets, Information sciences 177.1 (2007), pp. 327.

[14] A. Skowron, J. Stepaniuk: Tolerance approximation spaces, Fundamenta Informaticae 27.2 (1996), pp. 245-253.

[15] Y. YAO, B. YAO: Covering based rough set approximations, Information Sciences 200 (2012), pp. 91-107, ISSN: 0020-0255,

DOI: http://dx.doi.org/10.1016/j.ins.2012.02.065,

URL: http://www.sciencedirect.com/science/article/pii/S0020025512001934. 